\title{
Control de calidad y análisis fisicoquímicos en la cadena productiva de la leche de la parroquia EI Triunfo del cantón Pastaza
}

Quality control and physicochemical analysis in the milk production chain of the El Triunfo parish in the Pastaza canton

Sandra Gabriela Barrazueta Rojas. ${ }^{1}$, Ronny Alexander Quintana Unda. ${ }^{2}$, Guillermo Xavier Mendoza Zurita. ${ }^{3}$ \& Julio Mauricio Oleas López. ${ }^{4}$

\section{Recibido: 25-06-2019 / Revisado: 30-07-209 /Aceptado: 10-08-2019/ Publicado: 10-09-2019}

\begin{abstract}
DOI: https://doi.org/10.33262/cienciadigital.v3i3.4.838

Milk is a staple in the diet of consumers, which makes this food one of the most consumed. Ecuador produces 5'100,000 liters of milk daily and the eastern region has $8.79 \%$ of total production. Currently, collection centers have been implemented in the province of Pastaza with the objective of encouraging the production of quality milk. For this reason, the purpose of this work was to determine the quality of milk in the production chain of the collection center of El Triunfo parish in Pastaza province, for this purpose samples were taken from 33 active producers in the area; For the collection of the information, the quantity of production, milking conditions and transport were taken into account, for this, it was supported by the Pastaza MAGAP. The analysis of the samples was carried out in the laboratory of the collection center where the physical-chemical and bromatological parameters of the
\end{abstract}

\footnotetext{
${ }^{1}$ Escuela Superior Politécnica de Chimborazo, Facultad de Informática y Electrónica, Chimborazo, Ecuador, sbarrazueta@espoch.edu.ec

2 Unidad Educativa Comunitaria Intercultural Bilingüe San Jacinto, Pastaza, Ecuador, undalaexander@gmail.com

3 Avícola Don Guillo, Chambo, Ecuador, guillomendoza01@ yahoo.com

4 Escuela Superior Politécnica de Chimborazo, Facultad de Ciencias Pecuarias, Chimborazo, Ecuador, joleas@espoch.edu.ec
} 
milk were analyzed according to the INEN 9: 2012 standard. The variables evaluated were: density, $\mathrm{pH}$, temperature, fat, titratable acidity, total solids, non-fatty solids, proteins, protein stability, cryoscopy point and mastitis. The results obtained indicated that the milk of the El Triunfo parish collection center is mostly within the parameters established by the standard, but there are samples that are outside the established ranges, in addition it was determined that the food, transport, environmental conditions and animal handling have an effect on milk quality.

Keywords: Quality control, physicochemical analysis, production chain.

\section{Resumen}

La leche es un alimento básico en la dieta de los consumidores, lo que convierte a este alimento en uno de los más consumidos. Ecuador produce 5’100.000 litros de leche diarios y la región oriente tiene el $8.79 \%$ del total de la producción. Actualmente se ha implementado centros de acopio en la provincia de Pastaza con el objetivo de incentivar a la producción de leche de calidad, por lo mencionado anteriormente el propósito de este trabajo fue determinar la calidad de la leche en la cadena productiva del centro de acopio de la parroquia el Triunfo de la provincia de Pastaza, para ello se tomaron muestras de 33 productores activos de la zona; para el levantamiento de la información se tomó en cuenta: cantidad de producción, condiciones del ordeño y transporte, para ello se contó con el apoyo del MAGAP de Pastaza. El análisis de las muestras se lo realizó en el laboratorio del centro de acopio donde se analizó los parámetros físico-químicos y bromatológicos de la leche según indica la norma INEN 9:2012. Las variables evaluadas fueron: densidad, pH, temperatura, materia grasa, acidez titulable, sólidos totales, sólidos no grasos, proteínas, estabilidad proteica, punto crioscópico y mastitis. Los resultados obtenidos indicaron que la leche del centro de acopio de la parroquia el Triunfo en su mayoría se encuentra dentro de los parámetros establecidos por la norma, pero existen muestras que están fuera de los rangos establecidos, además se determinó que la alimentación, el transporte, condiciones ambientales y manejo del animal tienen efecto sobre la calidad de la leche.

Palabras clave: Control de calidad, análisis fisicoquímicos, cadena productiva. 


\section{INTRODUCCIÓN}

En Ecuador la producción lechera es una fuente de ingresos para los productores y provee de un alimento básico para la alimentación humana, los datos del censo agropecuario del año 2010 indican que la producción lechera se ha concentrado en la región de la Sierra, donde se encuentran un $73 \%$ de la producción nacional, $19 \%$ en la Costa y un $8 \%$ la Amazonía y las Islas Galápagos (AGSO, 2012). En el país existe una política de fomento ganadero soportada por un programa de capacitación, transferencia e innovación tecnológica apropiada, con lo que se ha duplicado fácilmente la producción de carne y leche de una forma sustentable en poco tiempo, ya que ha estimulado el consumo interno y se está participando en los mercados internacionales (Grijalva, J., 2014). Hoy en día las explotaciones lecheras aspiran a salvaguardar la salubridad y calidad de la leche cruda de forma que satisfaga las más altas expectativas de la industria alimentaria y de los consumidores. Las prácticas en la explotación deben también asegurar que la leche sea producida por animales sanos, bajo condiciones aceptables para estos últimos y en equilibrio con el entorno medio ambiental local. Los ganaderos, los proveedores de las explotaciones lecheras, transportistas, fabricantes de alimentos lácteos, distribuidores y minoristas deben ser parte integral del sistema de gestión de calidad y seguridad alimentaria. El mejoramiento de la calidad de la leche y de los productos derivados de la misma se ha convertido en uno de los objetivos primordiales en la industria alimentaria, las normas del Instituto Ecuatoriano de Normalización (INEN) presentan ciertas condiciones o características que debe cumplir un alimento para el consumo humano. Uno de los aspectos que se están desarrollando actualmente en el Plan de Desarrollo y Ordenamiento Territorial (PDOT) de la parroquia el Triunfo, respecto al área de manejo económico y productivo, está basado en mejorar, capacitar, dotar de infraestructura, equipos a los pequeños y medianos productores de la zona. El Ministerio de Agricultura Ganadería Acuicultura y Pesca (MAGAP) de la provincia de Pastaza y la asociación de productores de leche de la parroquia el Triunfo tienen la necesidad de conocer la calidad de leche que se está produciendo en el sector, con el fin de mejorar la cadena productiva de la leche corrigiendo aquellas fallas o malas prácticas que se aplican en el proceso, de acuerdo a las normativas vigente en Ecuador. Es por ello que mediante la presente investigación se realizará un levantamiento de información de la calidad de la leche que se recibe en el centro de acopio de la parroquia el Triunfo, así como 
los factores de mayor incidencia que afectan a la misma. Además, se capacitará técnicamente al personal del centro de acopio (técnicas de laboratorio, BPM, POES y normas INEN), transportistas (BPM, pruebas de anden) y productores (BPO y normas INEN). (AGSO, 2012) (FAO, 2011) (Codex Alimentarius, 2011).

\section{MATERIALES Y MÉTODOS}

\section{a. Localización y duración del experimento}

La presente investigación se realizó en la provincia de Pastaza en la parroquia El Triunfo, ubicada en el km 27 vía Arajuno con una extensión de $218 \mathrm{Km}^{2}$ y una altitud de 1073 m.s.n.m, según el censo de población del 2001, realizado por el INEC, la población de la parroquia El Triunfo es de 1,381 habitantes, .de los cuales 807 son hombres y 574 son mujeres (GAD Parroquial Rural el Triunfo, 2000).

El estudio tuvo una duración de 120 días, distribuidos en las actividades mencionadas en el cronograma de actividades que son: elaboración de registros de las condiciones de producción, recepción y calidad de la leche, recogida de muestras en granja, análisis físicoquímico de las muestras, capacitación de los proveedores y empleados del centro de acopio.

\section{b. Unidades experimentales}

Este trabajo tomó como muestra a 33 proveedores ubicados en la misma zona que entregan la producción en el centro de acopio el Triunfo sin restricción, debido a que se recopiló información de cada uno de ellos y se aplicó pruebas físico químicas y bromatológicas en las muestras recogidas.

\section{c. Instalaciones, equipos, materiales y reactivos}

Las instalaciones, equipos y materiales que se utilizaron en el presente trabajo son: implementos personales, cámara fotográfica, fichas técnicas, tubos de ensayo, vasos de precipitación, envases para muestras, homogenización de leche, pipetas, termómetros, acidómetro, master Eco y termolactodensímetro.

\section{d. Tratamientos y Diseño experimental}

Por ser un estudio a nivel de campo con respecto al levantamiento de información y a la calidad en el centro de acopio de la parroquia El Triunfo se consideraron los productores como unidades en la cadena productiva del centro de acopio. El estudio se basó en un levantamiento de datos y diagnóstico técnico mediante la aplicación de una distribución de frecuencias, la misma que fue elaborada y aplicada para identificar los efectos que puedan 
afectar a la calidad de la leche, y posteriormente recomendar y corregir aquellos puntos críticos que se hayan encontrado.

\section{e. Mediciones experimentales}

Se realizó pruebas físico-químicas (densidad, acidez titulable, estabilidad de etanol, reductasa y punto crioscópico), y pruebas bromatológicas (grasa, solidos no grasos, solidos totales, proteína, lactosa y agua añadida) (INEN, 2012).

\section{f. Análisis estadísticos y Prueba de significancia}

Se trabajó con un análisis descriptivo, para los parámetros de calidad de la leche, se realizó una distribución de frecuencias para determinar el efecto de la localización, altitud y otros parámetros que puedan afectar a la calidad de la leche. Se analizó mediante un ADEVA multifactorial los efectos del tipo de alimentación y el número total de animales. Con otro ADEVA multifactorial los efectos del transporte y recipientes utilizados para la leche y un tercer ADEVA unifactorial para analizar las diferencias entre productores. Finalmente, se realizó un ADEVA de medidas repetidas para evaluar el efecto del transporte sobre los parámetros de calidad de la leche.

\section{g. Procedimiento experimental}

En el presente estudio se aplicó una investigación de carácter descriptivo, se recopilaron datos de los procesos y actividades que realizan los trabajadores y proveedores del centro de acopio de la parroquia el Triunfo, los mismos que fueron analizados para determinar el riesgo que estos causan a la calidad de la leche y proponer posibles soluciones.

\section{h. Metodología de evaluación}

Se determinó la calidad de la leche en el centro de acopio el Triunfo por medio de pruebas físico químicas y bromatológicas, además de la recolección de datos en toda la cadena productiva de cada uno de los productores de la leche, con el fin de levantar información básica de la calidad de la leche, es por eso que se brindó capacitación técnica a los proveedores y empleados del centro de acopio en lo referente a BPO y técnicas de laboratorio (INEN, 2012).

\section{RESULTADOS Y DISCUSIÓN}

\section{Primera ficha de información}

Se detalla las condiciones de producción de leche en la parroquia El Triunfo": proveedores, número de animales, raza, producción de leche, animales en ordeño, sistema de alimentación 
(pastoreo y balanceado) y transporte (propio o intermediario, bidones o recipientes de plástico). Se realizaron encuestas a cada uno de los productores activos y por medio de visitas planificadas se comprobó la veracidad de la información obtenida.

\section{Segunda ficha de información}

El segundo levantamiento de información fue a pie de finca. Se recogió información sobre las condiciones de producción de la leche: la limpieza de las ubres, materiales de limpieza, tipo de agua utilizada en el ordeño, el tipo de suelo donde se realiza el ordeño, limpieza del ordeñador, utensilios y condición del animal. Esta ficha se levantó en 19 proveedores, debido a que no fue posible realizar la visita de campo a los 33 proveedores.

\section{Calidad de leche por productores}

Se detallan las medias obtenidas de la calidad de la leche por productor. Se encontraron diferencias significativas $(\mathrm{P}<0,05)$ para todos los parámetros evaluados; estas diferencias pueden ser debidas a múltiples factores como: raza de los animales, condiciones de ordeño, transporte, limpieza, distancia al centro de acopio, cantidad de leche producida, condiciones ambientales y adulteración de la leche (Calderón A., 2006).

\section{Efecto de la alimentación y el número de animales en la calidad de leche}

A continuación, se detallan las medias obtenidas para la calidad de la leche referente a la interrelación de los efectos alimentación y número de animales. Se encontraron diferencias significativas $(\mathrm{P}<0,05)$, en las variables analizadas; pero debido a que la alimentación y el animal están relacionados directamente con la composición nutricional de la leche solo se tomara en cuenta las siguientes variables: sólidos grasos, no grasos, sólidos totales, proteína, lactosa y volumen de producción. Estas diferencias pueden ser debidas a múltiples factores como: raza de los animales, porcentaje y concentración de balanceado, calidad del forraje, condiciones ambientales y adulteración de la leche (Kukilinski, C. 2003) (Malchiodi, F. et al., 2014). El número de animales afectó para los sólidos grasos en los animales que comían balanceado diariamente, siendo las explotaciones con más animales las que presentaron un menor valor de grasa en leche (3,34\%). En los sólidos no grasos se encontró que existen diferencias significativas en la calidad de leche de los animales que recibían el aporte de balanceado pasando un día donde las granjas de 6-10 animales obtuvieron los menores valores. Estas diferencias significativas se deben a la concentración de balanceado no es la 
misma para todos los animales, ya que en una explotación con mayor número de animales el aporte de balanceado es menor en cantidad (Holt, C. et, al 1988).

También hay que tener en cuenta que la alimentación, en general, de los animales es muy irregular y que este estudio está hecho en una época del año concreta (Owen, E. et al., 2005). Con respecto a la variable de solidos totales en el efecto del aporte de balanceado las vacas de las explotaciones de 11 o más animales presentaron menor concentración de solidos totales cuando eran alimentadas con balanceado diariamente. Por otro lado, en las explotaciones en las que se alimentaba con balanceado pasando un y presentaban 11 o más animales mostraron valores superiores de sólidos totales (12,67 \%) (Farrell, H. et al., 2004). Para las variables punto crioscópico y lactosa no se encontró diferencias significativas para ninguno de los efectos aplicados.

Con respecto a la proteína, el aporte de balanceado no tuvo un efecto significativo sobre esta variable, en cuanto al número de animales, aquellas explotaciones que daban balanceado pasando un día y cuentan con 6 a 10 animales presentaron un valor inferior 2,98\%.

Esto puede ser debido a factores que se relacionan con el número de animales, pero que no han sido tomados en cuenta para esta investigación (Ginger, M. y Grigor, M. 1999).

En el volumen encontramos diferencias significativas, donde el efecto del aporte de balaceado de vez en cuando con número de animales de 11 o más presento un valor de $75 \mathrm{~L}$, esto se debe a que existen productores que disponen de mayor cantidad animales a diferencia de otros que poseen menor número de animales, además que la alimentación no es la misma para cada una de las explotaciones (tabla 1).

Tabla 1. Efecto de la alimentación y el número de animales en la calidad de leche.

\begin{tabular}{|c|c|c|c|c|c|c|c|c|c|c|c|c|c|}
\hline \multicolumn{2}{|c|}{$\begin{array}{l}\text { Aporte de } \\
\text { balanceado }\end{array}$} & \multirow[t]{2}{*}{ Animales } & Temperatura ${ }^{\circ} \mathrm{C}$ & $\begin{array}{c}\text { Densidad } \\
\mathrm{g} / \mathrm{cm}^{3}\end{array}$ & $\begin{array}{c}\text { Acidez } \\
{ }^{\circ} \mathrm{D}\end{array}$ & $\mathrm{Ph}$ & \begin{tabular}{|c|} 
Grasa \\
$\%$
\end{tabular} & $\begin{array}{c}\text { Solidos no } \\
\text { grasos \% }\end{array}$ & $\begin{array}{l}\text { Solidos } \\
\text { totales } \%\end{array}$ & $\begin{array}{c}\text { Punto } \\
\text { crioscópico }{ }^{\circ} \mathrm{C}\end{array}$ & \begin{tabular}{|c|} 
Proteina \\
$\%$
\end{tabular} & Lactosa $\%$ & \begin{tabular}{|c|} 
Volumen \\
L
\end{tabular} \\
\hline \multirow{2}{*}{ Diaria } & 0.5 & & $29,16 \mathrm{~b}$ & $1,028 \mathrm{a}$ & $16,27 \mathrm{~B}$ & $6,7 \mathrm{ab}$ & $4,01 \mathrm{~A}$ & $8,4 \mathrm{Ab}$ & $12,41 \mathrm{ab}$ & $0,539 \mathrm{a}$ & $3,05 \mathrm{a}$ & $4,36 \mathrm{a}$ & $25,28 \mathrm{~b}$ \\
\hline & $11-0$ & & 30,76 a & 1,028 a & $15,48 \mathrm{~B}$ & $6,7 \mathrm{ab}$ & $3,34 \mathrm{~B}$ & $8,21 \mathrm{~B}$ & $11,54 \mathrm{~b}$ & $0,52 \mathrm{a}$ & $3,02 \mathrm{ab}$ & $4,41 \mathrm{a}$ & $39,56 \mathrm{~b}$ \\
\hline Pasando & 0.5 & & $29,43 \mathrm{~b}$ & 1,028 a & $16,6 \mathrm{Ab}$ & $6,7 \mathrm{ab}$ & $3,88 \mathrm{~A}$ & $8,31 \mathrm{ab}$ & $12,19 \mathrm{ab}$ & $0,54 \mathrm{a}$ & $3,07 \mathrm{a}$ & $4,4 \mathrm{a}$ & $45,91 \mathrm{~b}$ \\
\hline un dia & & 06-oct & $29,68 \mathrm{~b}$ & $1,028 \mathrm{a}$ & $16,28 \mathrm{Ab}$ & $6,7 \mathrm{~b}$ & $4,02 \mathrm{~A}$ & $8,13 \mathrm{~B}$ & $12,14 \mathrm{~b}$ & $0,516 \mathrm{a}$ & $2,98 \mathrm{~b}$ & $4,41 \mathrm{a}$ & $40,06 \mathrm{~b}$ \\
\hline \multirow[t]{3}{*}{$A$ veces } & & 06-oct & $30,29 \mathrm{ab}$ & $1,027 \mathrm{~b}$ & $17,57 \mathrm{~A}$ & $6,8 \mathrm{a}$ & $4,23 \mathrm{~A}$ & $8,29 \mathrm{ab}$ & $12,52 \mathrm{ab}$ & 0,531 a & $3 a b$ & $4,44 \mathrm{a}$ & $58,14 \mathrm{ab}$ \\
\hline & $11-0$ & mas & $28,71 \mathrm{~b}$ & $1,027 \mathrm{~b}$ & $17,43 \mathrm{~A}$ & $6,7 \mathrm{a}$ & $4,23 \mathrm{~A}$ & $8,08 \mathrm{~B}$ & $12,3 \mathrm{ab}$ & 0,528 a & $3 a b$ & $4,26 \mathrm{a}$ & $75 \mathrm{a}$ \\
\hline & EEM & & 0,131 & 0,34 & 0,08 & 0 & 0,03 & 0,003 & 0,057 & 0,003 & 0,012 & 0,019 & 1,324 \\
\hline
\end{tabular}

$(\mathrm{a}, \mathrm{b})$ valores que no comparten la misma letra dentro de cada columna difieren estadísticamente $(\mathrm{P} \leq 0,05)$. 


\section{Efecto del transporte y los recipientes utilizados por los proveedores en la calidad de la leche.}

En la tabla 2, se detallan las medias obtenidas de la calidad de la leche referente al efecto del transporte y los recipientes utilizados. Se encontraron diferencias significativas $(\mathrm{P}<0,05)$, en las variables analizadas. Pero debido a que el transporte y los recipientes no están relacionados directamente con la composición nutricional de la leche solo se tomara en cuenta las siguientes variables: temperatura, acidez, densidad y $\mathrm{pH}$.

La norma INEN (9:2012) menciona que la leche después de haber sido obtenida del ordeño debe ser enfriada, almacenada y transportada hasta el centro de acopio en recipientes apropiados autorizados por las autoridades sanitarias competentes, donde debe ser filtrada, enfriada y almacenada a una temperatura inferior a $10{ }^{\circ} \mathrm{C}$ con agitación constante. En el efecto del transporte y los recipientes se encontró que hay diferencias significativas en la variable temperatura. Cuando el transporte es propio y se utiliza bidones de acero se observó la mayor temperatura $\left(30,31^{\circ} \mathrm{C}\right)$. Este valor pudo darse porque los proveedores que disponen de su propio vehículo, no tienen la urgencia de entregar a horas tempanas la leche en el centro de acopio, y debido a las condiciones ambientales la leche disminuye su temperatura (Codex Alimentarius, 2011).

Para la variable densidad no se encontraron diferencias significativas con el efecto del transporte y los recipientes utilizados. Para la acidez se encontraron diferencias significativas para el efecto del transporte, indicando que aquellos que participan del intermediario presentan los valores más altos de acidez $\left(16,50-17,32^{\circ} \mathrm{D}\right)$ a diferencia de los que transportan la leche en sus propios vehículos $\left(15,57-15,9^{\circ} \mathrm{D}\right)$. Respecto al efecto del recipiente no se encontró efecto del uso de diferentes materiales para el transporte. El intermediario no posee las condiciones para realizar el transporte de la leche ya que el producto está en contacto con el ambiente y pasa por determinadas horas expuestas a las condiciones climáticas (Revilla, A. 2008). 
Tabla 2. Efecto del transporte y los recipientes utilizados por los proveedores en la calidad de leche.

\begin{tabular}{|c|c|c|c|c|c|c|c|c|c|c|c|c|}
\hline Transporte & Recipientes & $\begin{array}{c}\text { Temperatura } \\
{ }^{\circ} \mathrm{C}\end{array}$ & $\begin{array}{l}\text { Densidad } \\
\mathrm{g} / \mathrm{cm}^{3}\end{array}$ & Acidez ${ }^{\circ} \mathrm{D}$ & $\mathrm{pH}$ & Grasa \% & $\begin{array}{l}\text { Solidos no } \\
\text { grasos \% }\end{array}$ & $\begin{array}{l}\text { Solidos } \\
\text { totales \% }\end{array}$ & $\begin{array}{c}\text { Punto } \\
\text { crioscópico } \\
\%\end{array}$ & $\begin{array}{c}\text { Proteina } \\
\%\end{array}$ & $\begin{array}{c}\text { Lactosa } \\
\%\end{array}$ & $\begin{array}{c}\text { Volumen } \\
\%\end{array}$ \\
\hline \multirow{3}{*}{ Propio } & Cantara & 30,31 a & 1,028 & $16,38 \mathrm{~b}$ & $6,7 \mathrm{~b}$ & $3,89 \mathrm{~b}$ & $8,29 \mathrm{~b}$ & $12,18 \mathrm{~b}$ & $0,527 \mathrm{a}$ & $3,04 a b$ & $4,35 \mathrm{a}$ & $37,77 \mathrm{~b}$ \\
\hline & Plástico & $29,29 \mathrm{~b}$ & 1,028 & $15,57 \mathrm{~b}$ & $6,74 a b$ & $3,99 \mathrm{ab}$ & $8,66 \mathrm{a}$ & $12,64 a b$ & 0,554 a & $3,13 \mathrm{a}$ & $4,56 \mathrm{a}$ & $72,86 \mathrm{a}$ \\
\hline & Mixto & $29,36 \mathrm{~b}$ & 1,028 & $15,99 \mathrm{~b}$ & $6,72 \mathrm{~b}$ & $3,8 \mathrm{~b}$ & $8,28 \mathrm{~b}$ & $12,08 \mathrm{~b}$ & $0,524 \mathrm{ab}$ & $3,03 \mathrm{ab}$ & $4,38 \mathrm{a}$ & $37,68 \mathrm{~b}$ \\
\hline \multirow{4}{*}{ Intermediario } & Cantara & $29,74 \mathrm{ab}$ & 1,028 & $17,32 \mathrm{a}$ & $6,8 \mathrm{a}$ & $3,88 \mathrm{~b}$ & $8,21 \mathrm{~b}$ & $12,09 \mathrm{~b}$ & $0,527 \mathrm{ab}$ & $3 b$ & $4,36 \mathrm{a}$ & $57,29 \mathrm{a}$ \\
\hline & Plástico & $29,45 \mathrm{~b}$ & 1,028 & 17,08 a & $6,73 \mathrm{~b}$ & $4,19 \mathrm{a}$ & $8,48 \mathrm{a}$ & $12,67 \mathrm{a}$ & 0,554 a & $3,1 \mathrm{a}$ & $4,42 \mathrm{a}$ & $39,41 \mathrm{~b}$ \\
\hline & Mixto & $29 \mathrm{~b}$ & 1,027 & $16,5 \mathrm{ab}$ & $6,7 \mathrm{~b}$ & $3,86 \mathrm{~b}$ & $7,82 \mathrm{~b}$ & $11,68 \mathrm{~b}$ & $0,494 \mathrm{~b}$ & $2,85 \mathrm{~b}$ & $4,14 \mathrm{~b}$ & $67,29 \mathrm{a}$ \\
\hline & EEM & 0,131 & 0,004 & 0,086 & 0,023 & 0,032 & 0,033 & 0,057 & 0,003 & 0,012 & 0,019 & 1,324 \\
\hline
\end{tabular}

$(\mathrm{a}, \mathrm{b})$ valores que no comparten la misma letra dentro de cada columna difieren estadísticamente $(\mathrm{P} \leq 0,05)$.

\section{Efecto de comparación respecto al transporte de la leche desde la finca hasta el centro de acopio.}

Para el efecto del transporte sobre la calidad de la leche se realizó la comparación de las muestras recogidas tanto a pie de finca como a la llegada del centro de acopio (tabla 3), se encontraron diferencias significativas para todas las variables (menos el $\mathrm{pH}$ ). Para la temperatura se puede observar que durante el transporte se consigue una reducción de aproximadamente $3^{\circ} \mathrm{C}$, lo cual es insuficiente para el óptimo transporte de la leche. Posiblemente debido al transporte con estas temperaturas se observa un incremento de la acidez cuando la leche llega al centro de acopio. Con respecto a los parámetros nutricionales se encontró un aumento de los mismos durante el transporte, estos incrementos podrían ser debidos a que la toma de muestras en el centro de acopio se realizó sin la debida agitación, por lo que se debe mejorar la técnica empleada (MAGAP, 2012).

Tabla 3. Efecto de comparación respecto al transporte de la leche desde la finca hasta el centro de acopio.

\begin{tabular}{|l|c|c|c|c|c|}
\hline Variables & Finca & & Acopio & & Error estándar \\
\hline Temperatura & 32,58 & $\mathrm{~A}$ & 29,21 & $\mathrm{~b}$ & 0,347 \\
\hline Densidad & 1,026 & $\mathrm{~A}$ & 1,028 & $\mathrm{~b}$ & 0 \\
\hline Acidez & 15,37 & $\mathrm{~A}$ & 16,37 & $\mathrm{~b}$ & 0,178 \\
\hline $\mathrm{pH}$ & 6,7 & $\mathrm{~A}$ & 6,7 & $\mathrm{a}$ & 0 \\
\hline Grasa & 3,46 & $\mathrm{~A}$ & 3,85 & $\mathrm{~b}$ & 0,076 \\
\hline Solidos no grasos & 7,91 & $\mathrm{~A}$ & 8,31 & $\mathrm{~b}$ & 0,057 \\
\hline Punto crioscópico & 0,5 & $\mathrm{~A}$ & 0,522 & $\mathrm{~b}$ & 0,044 \\
\hline Proteina & 2,91 & $\mathrm{~A}$ & 3,04 & $\mathrm{~b}$ & 0,023 \\
\hline Lactosa & 4,2 & $\mathrm{~A}$ & 4,4 & $\mathrm{~b}$ & 0,031 \\
\hline
\end{tabular}

$(\mathrm{a}, \mathrm{b})$ valores que no comparten la misma letra dentro de cada columna difieren estadísticamente $(\mathrm{P} \leq 0,05)$. 


\section{CONCLUSIONES}

Se determinó que la mayoría de las muestras recogidas en el centro de acopio se encuentran dentro de las especificaciones de la norma INEN, respecto a los requisitos físico-químicos y bromatológicos, y en menor cantidad ciertas muestras están fuera de los parámetros, esto es debido a que no se dispone en el sector de la tecnología cadena de frío y las condiciones de producción.

La calidad de la leche de la parroquia el Triunfo mostro una gran variación en los parámetros físico-químicos como bromatológicos, entre los productores de la zona, dando como media a la leche del centro de acopio los siguientes valores: Temperatura $32,58{ }^{\circ} \mathrm{C}$, Densidad 1,026 $\mathrm{g} / \mathrm{cm}^{3}$, Acidez $15,37^{\circ} \mathrm{D}$, pH 6,7, grasa 3,46\%, solidos no grasos 7,91\%, punto crioscópico $0,5^{\circ} \mathrm{C}$, proteína $2,91 \%$ y lactosa $4,2 \%$.

Los puntos críticos identificados en la cadena productiva de la leche de la parroquia el Triunfo fueron: manejo del ordeño (limpieza de la ubre, materiales y limpieza de los utensilios), transporte (recipientes empleados, falta de sistema de refrigeración, tiempo transcurrido desde el ordeño hasta la llegada al centro de acopio) y centro de acopio (limpieza de los equipos y materiales).

\section{Referencias}

1. AGSO Asociación de Ganaderos de la Sierra y Oriente. (2012). Producción de leche en el Ecuador. http://www.agso.com.ec.

2. Calderón, A. (2006). Indicadores de la calidad de leches crudas en diferentes regiones de Colombia. Pp.3.

3. CODEX ALIMENTARIUS SEGUNDA EDICION (2011). Términos Generales (CODEX STAN 206 1999).

4. CODEX ALIMENTARIUS SEGUNDA EDICION. (2011) código de prácticas de higiene para la leche y los productos lácteos (CAC/RCP 57- 2004).

5. FAO. (2011). Cadena de calidad de la leche. Disponible en http://www.fao.org/ag/AGAinfo/subjects/es/dairy/ quality_chain.html.

6. FAO. (2011). Producción y Sanidad Animal Situación de la Lechería en América Latina y el Caribe. 
7. Farrell H. M. R. Jiménez-Flores, G. T. Bleck, E. M. Brown, J. E. Butler, Faye, B. Y Konuspayeva, G. (2012). The sustainability challenge to the dairy sector- The growing importance of non-cattle milk production worldwide. International Dairy Journal, 24:50-56.

8. GAD Parroquial Rural el Triunfo, (2000). Plan de Desarrollo y Ordenamiento Territorial. Registro oficial No 193.

9. Ginger, M.R. y Grigor, M.R. (1999). Comparative aspects of milk caseins. Review. Comparative Biochemistry and Physiology. 124:133-145.

10. Grijalva, J. (2014). Universidad de San Francisco de Quito, sistema innovador para procesos de producción y comercialización de la leche en Ecuador.

11. Holt C, Sawyer L. (1988). Primary and predicted secondary structuresof the caseins in relation to their biological functions. Protein Eng 2:251-9.

12. INEN Instituto Ecuatoriano de Normalización. (2012). Leche cruda requisitos. Norma INEN NTE 9:2012. 3 rev. Quito, EC.

13. Kukilinski, C. (2003). Nutrición y Bromatología, Chile: Edición Omega pp.213-216.

14. MAGAP (2012). Guía de buenas prácticas pecuarias de producción de leche. resolución técnica No 0217.

15. Malchiodi, F., Cecchinato, A., Penasa, M., Cipolat-Gotet, C., Bittante, G. (2014). Milk quality, coagulation properties, and curd firmness modeling of purebred Holsteins and first- and secondgeneration crossbred cows from Swedish Red, Montbéliarde, and Brown Swiss bulls. Journal of Dairy Science, 97:4530-4541.

16. Owen, E., Kitalyi, A., Jayasuriya, N., Y Smith, T. (2005). Livestock and wealth creation: improving the husbandry of animals kept by resourcepoor people in developing.

17. Revilla, A. (1996). Tecnología de La Leche. Tercera edición. Escuela Agrícola Panamericana Honduras. Pp.396.

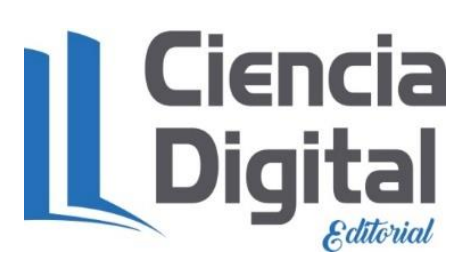




\section{PARA CITAR EL ARTÍCULO INDEXADO.}

Barrazueta Rojas, S., Quintana Unda, R., Mendoza Zurita, G., \& Oleas López, J. (2019). Control de calidad y análisis fisicoquímicos en la cadena productiva de la leche de la parroquia El Triunfo del cantón Pastaza. Ciencia Digital,3(3.4.), 104-115. https://doi.org/10.33262/cienciadigital.v3i3.4.838

\section{Ciencia \\ Ligital \\ Editorial}

El artículo que se publica es de exclusiva responsabilidad de los autores y no necesariamente reflejan el pensamiento de la Revista Ciencia Digital.

El artículo queda en propiedad de la revista y, por tanto, su publicación parcial y/o total en otro medio tiene que ser autorizado por el director de la Revista Ciencia Digital.
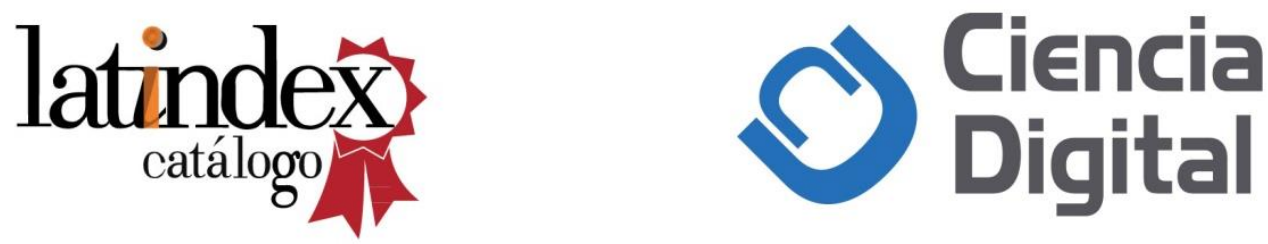